\title{
Pulsating pre-Main Sequence stars in young open clusters
}

\author{
Konstanze Zwintz ${ }^{1}$, Marcella Marconi ${ }^{2}$, Thomas Kallinger ${ }^{1}$ \\ and Werner W.Weiss ${ }^{1}$ \\ ${ }^{1}$ Institute of Astronomy, University of Vienna, Türkenschanzstrasse 17, A-1180 Vienna, \\ Austria \\ email: zwintz@astro.univie.ac.at \\ ${ }^{2}$ INAF Astronomical Observatory of Capodimonte, Naples, Italy
}

\begin{abstract}
New pulsating pre-main sequence (PMS) stars have been discovered in the young open clusters IC 4996 and NGC 6383 using CCD time series photometry in Johnson $B$ and $V$ filters. As the cluster ages are both smaller than 10 million years, all members later than spectral type A0 are still contracting towards the ZAMS, hence providing ideal candidates for searches of pulsation. A dozen stars in NGC 6383 and 35 stars in IC 4996 lie within the boundaries of the classical instability region in the Hertzsprung-Russell (HR) diagram, but pulsation was detected for only two of them in each cluster.

For the well-studied cluster NGC 2264, the two already known PMS pulsating members, V 588 Mon and V 589 Mon, have been analysed using new data from a multi-site campaign. All data collected since their discovery in 1972 build the basis for the first measurements of period changes in PMS pulsators.
\end{abstract}

Keywords. Techniques: photometric, stars: pre-main-sequence, stars: variables: other, Galaxy: open clusters and associations: individual (NGC 6383, IC 4996)

\section{Introduction}

Pre-main sequence (PMS) stars are evolving from the birthline to the zero-age main sequence (ZAMS) in the HR-diagram. During this phase they are characterized by a high degree of activity. They display photometric and spectroscopic variabilities on time scales between a couple of minutes and several months. They sometimes strongly interact with the circumstellar environment, in which they are still embedded. Very often emission lines appear in their spectra and UV and/or IR excesses are present. As PMS stars cross the instability region while contracting towards the ZAMS, part of their activity is due to stellar pulsations. The time PMS stars spend in this phase is extremaly short. A 1.5 $M_{\odot}$ star crosses the instability region in $10^{6}$ years while a $4 M_{\odot}$ star needs only 80000 years. At present 24 PMS pulsators are known displaying periods between 18 minutes (Amado et al. 2004) and 6 hours.

\section{Evolutionary stage}

PMS stars differ from their more evolved counterparts of same temperature and luminosity only in their interior structure, while their envelope properties are quite similar. The determination of the evolutionary stage of a field star may be ambiguous as the evolutionary tracks for PMS and post-main sequence stars intersect each other several times (see Fig. 1, PMS and post-main sequence evolutionary tracks are taken from D'Antona \& Mazzitelli (1994) and Breger \& Pamyatnykh (1998), respectively). 


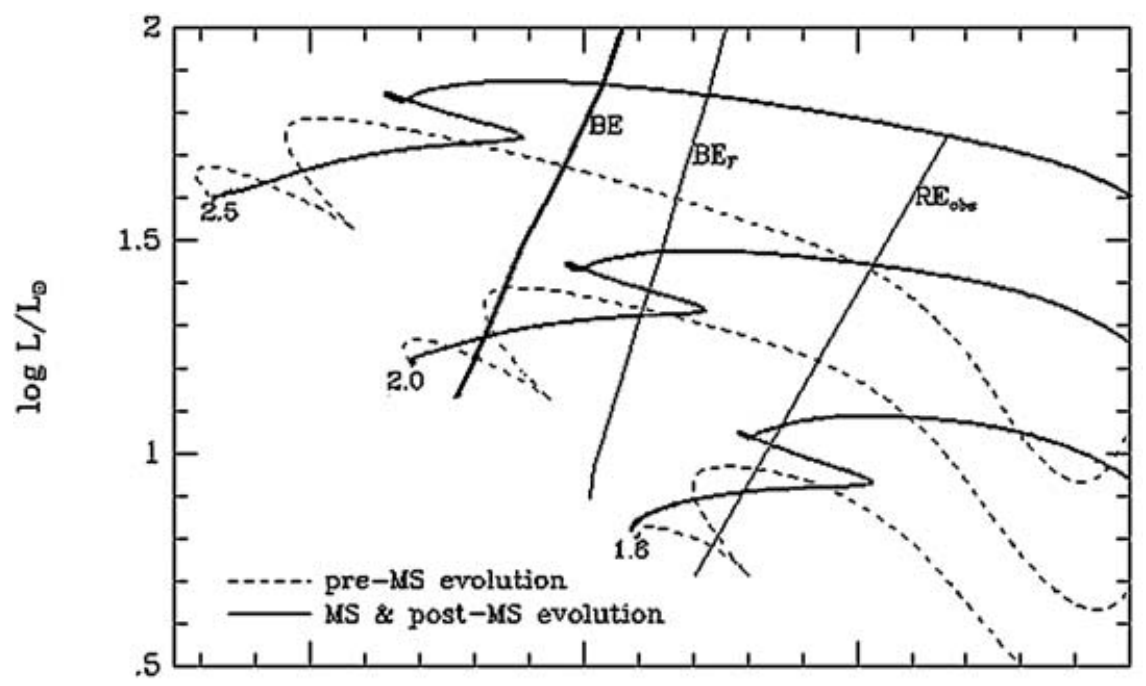

Figure 1. Intersecting pre- and post-main sequence evolutionary tracks for 1.6, 2.0 and $2.5 M_{\odot}$ and the boundaries of the classical instability strip, where $\mathrm{RE}_{\mathrm{obs}}$ denotes the empirical red edge, $\mathrm{BE}$ the blue edge for the radial overtones and $\mathrm{BE}_{\mathrm{F}}$ the blue edge for the fundamental mode (Breger \& Pamyatnykh 1998).

The study of young open clusters provides the possibility to reduce confusion with more evolved objects, as all members have the same age and distance. Clusters younger than a few $10^{7}$ yr show a main sequence down to $\sim$ B9, while cluster members of later spectral types have not yet reached the ZAMS. Hence, time series observations of the young open clusters NGC 6383 and IC 4996 were carried out to increase the number of pulsating PMS stars. New data from the PMS pulsators V 588 Mon and V 589 Mon in NGC 2264 were obtained during a multi-site campaign in 2002, and the pulsational characteristics of the stars were investigated in detail.

\section{NGC 6383}

NGC 6383 belongs to the Sgr OB1 association $\left(\alpha_{2000}=17^{h} 34.8^{m}, \delta_{2000}=-32^{\circ} 34^{\prime}\right)$ and has an age of $\sim 1.7 \mathrm{Myr}$. Its main sequence reaches only to B9, so cluster members of spectral types A - F are still in their PMS phase, hence are ideal candidates to search for pulsation. CCD time series photometry in Johnson $B$ and $V$ filters was obtained with the 0.9-m telescope of Cerro Tololo Interamerican Observatory (CTIO), Chile, within 9 nights. Two new PMS pulsators have been detected, NGC 6383170 and NGC 6383198.

\section{1. $N G C 6383170$}

For NGC 6383170 ( $V=12.61 \mathrm{mag}$ ), Thé et al. (1985) found $\mathrm{H}_{\alpha}$ in emission and high excess radiation in the NIR, typical for Herbig Ae/Be stars. With a spectral type of A5 IIIp (van den Ancker et al. 2000), a confirmed membership in NGC 6383, and a position above the ZAMS, NGC 6383170 is a newly discovered PMS pulsator (see Fig. 2). Five frequencies spanning a period range between 1.24 and 2.89 hours (see Table 1 ) have been detected (Zwintz et al. 2004).

Linear, nonadiabatic pulsation was calculated for radial modes of PMS models resulting in three possible solutions. No model reproduces all five frequencies simultaneously, but given the probable coexistence of radial and nonradial modes in these stars, not all frequencies need to correspond to radial pulsation. The model fitting the observed 


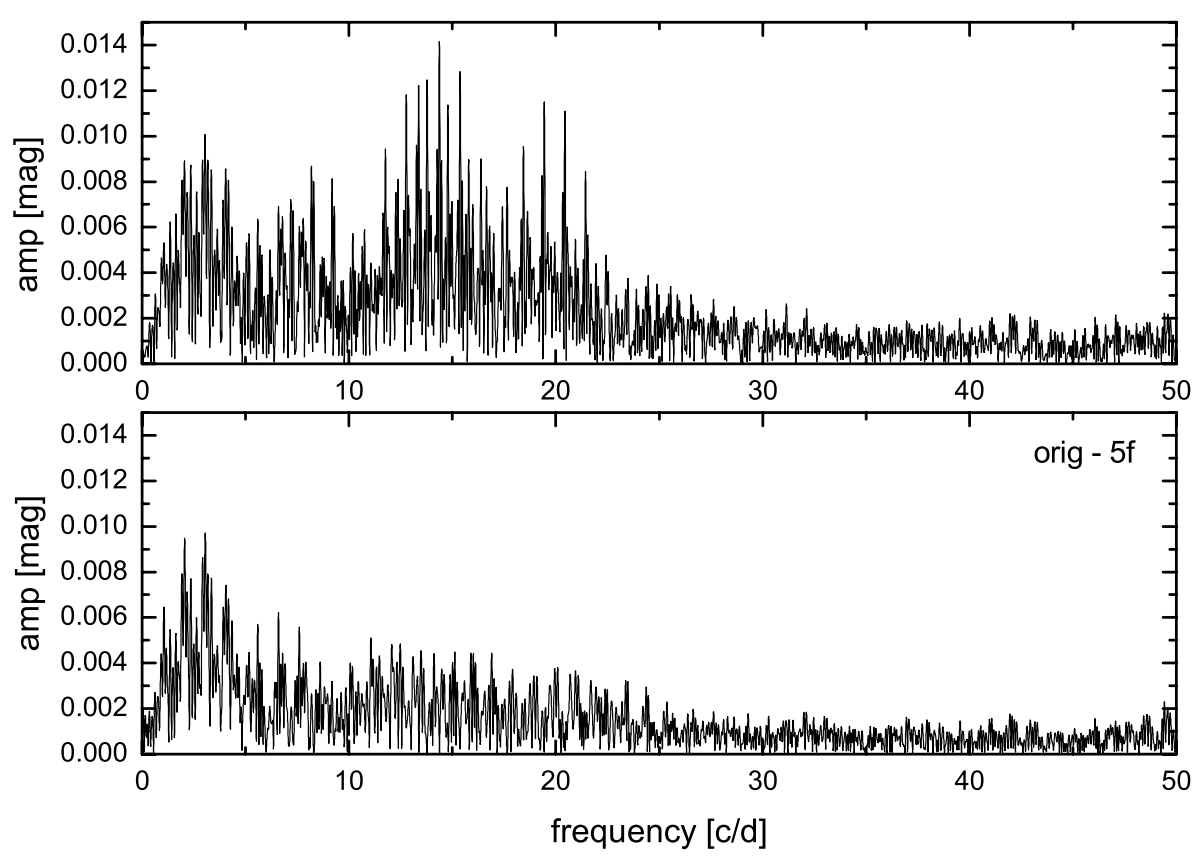

Figure 2. Amplitude spectra of NGC 6383170 in the $V$ filter; top: original data, bottom: after prewhitening the five significant frequencies.

frequencies best, gives a stellar mass of $2.5 M_{\odot}, \log L / L_{\odot}=1.68, T_{\text {eff }}=8100 \mathrm{~K}$, and pulsation in third $(f 1)$ and fifth overtones $(f 2)$. The solution seems to be optimal, because it is closest to the parameters derived spectroscopically by van den Ancker et al. (2000) (see Zwintz et al. 2004).

\section{2. $N G C 6383198$}

In the data of NGC 6383198 , only a single frequency of $19.024 \mathrm{~d}^{-1}$, corresponding to a period of $\sim 1.26$ hours, is significant in both filters (see Fig. 3 ).

Calculations of linear, nonadiabatic, radial pulsation models were also performed. As no spectral classification is available for this star, the $V$ and $(B-V)$ values were used to derive empirical ranges of luminosity and effective temperature based on the transformations given by Kenyon \& Hartmann (1995). Given the cluster membership of NGC 6383198 , it seems reasonable that the star pulsates with a single frequency in the third radial overtone having $2.0 M_{\odot}, \log L / L_{\odot}=1.3$ and $T_{\text {eff }}=7345 \mathrm{~K}$ (Zwintz et al. 2004).

\section{IC 4996}

IC 4996 belongs to the Cygnus star forming region $\left(\alpha_{2000}=20^{h} 16^{m} 34^{s}, \delta_{2000}=\right.$ $37^{\circ} 39^{\prime} 54^{\prime \prime}$ ) and is $\sim 7.5$ Myr old. CCD time series photometry in Johnson $B$ and $V$ filters was obtained using the 1.5-m telescope at Sierra Nevada Observatory (OSN), Spain, within 10 nights. Two new PMS pulsators IC 499637 and IC 499640 were discovered.

\section{1. $I C 499637$}

IC 499637 ( $V=15.30 \mathrm{mag}$ ) has a spectral type of A5 (Delgado et al. 1999) and pulsates with a single frequency corresponding to a period of $45.2 \mathrm{~min}$ (see Table 1) most probably in the $4^{\text {th }}$ radial overtone mode (Marconi 2004). 


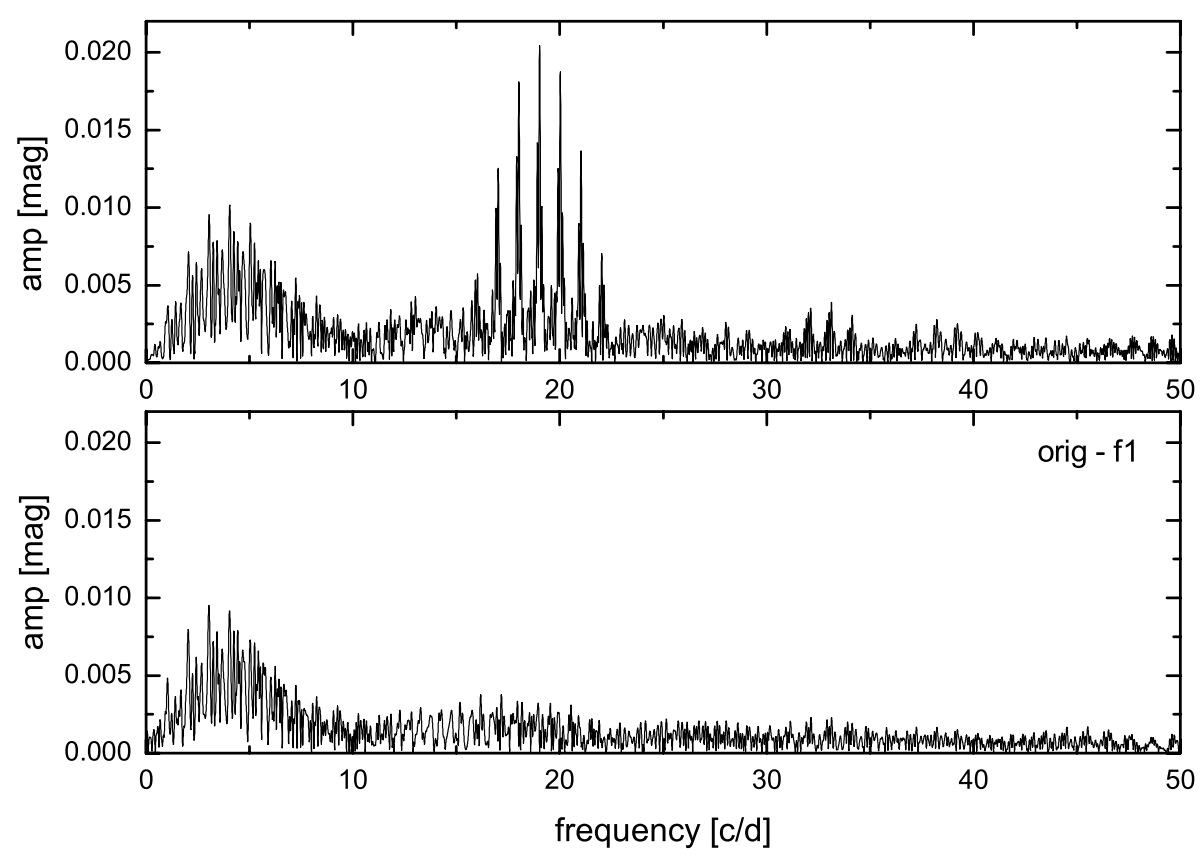

Figure 3. Amplitude spectra of NGC 6383198 in the $V$ filter; top: original data, bottom: after prewhitening the single significant frequency.

Table 1. Frequencies, amplitudes and phase shifts determined for the four new PMS pulsators NGC 6383 170, NGC 6383 198, IC 499637 and IC 499640.

\begin{tabular}{llrrrr}
\hline star no & & $\begin{array}{c}\text { frequency } \\
{\left[\mathrm{d}^{-1}\right]}\end{array}$ & $\begin{array}{r}V \text { amp. } \\
{[\mathrm{mmag}]}\end{array}$ & $\begin{array}{r}B \text { amp. } \\
{[\mathrm{mmag}]}\end{array}$ & \\
\hline NGC 6383 170 & $f 1$ & 14.376 & 12.5 & 16.0 & -0.243 \\
& $f 2$ & 19.436 & 11.3 & 14.9 & -0.092 \\
& $f 3$ & 13.766 & 9.8 & 12.3 & 0.474 \\
& $f 4$ & 8.295 & 8.6 & 11.1 & 0.035 \\
NGC 6383 198 & $f 5$ & 17.653 & 7.6 & 9.8 & -0.050 \\
& $f 1$ & 19.024 & 20.8 & 26.4 & 0.114 \\
\hline IC 4996 37 & $f 1$ & 31.875 & 4.6 & 4.6 & -0.200 \\
IC 4996 40 & $f 1$ & 33.569 & 7.6 & 8.5 & -0.138 \\
\hline
\end{tabular}

\section{2. $I C 499640$}

IC 499640 ( $V=15.03 \mathrm{mag}$ ) is of spectral class A4 (Delgado et al. 1999) and shows a single pulsation period of 42.9 min (see Table 1). Using linear, non-adiabatic, radial pulsation models, this period was identified as a $5^{\text {th }}$ radial overtone mode (Marconi 2004).

\section{NGC 2264}

The well-known cluster NGC 2264 is $\sim 8$ Myr old and contains the first two detected PMS $\delta$ Scuti-like stars, V 588 Mon (NGC 2264 2, HD 261331) and V 589 Mon (NGC 2264 20, HD 261446) investigated by Breger (1972). A multi-site campaign was carried out to reinvestigate the pulsational properties of the two stars. This resulted in the detection of a much denser frequency spectra than available for any other PMS pulsator. 


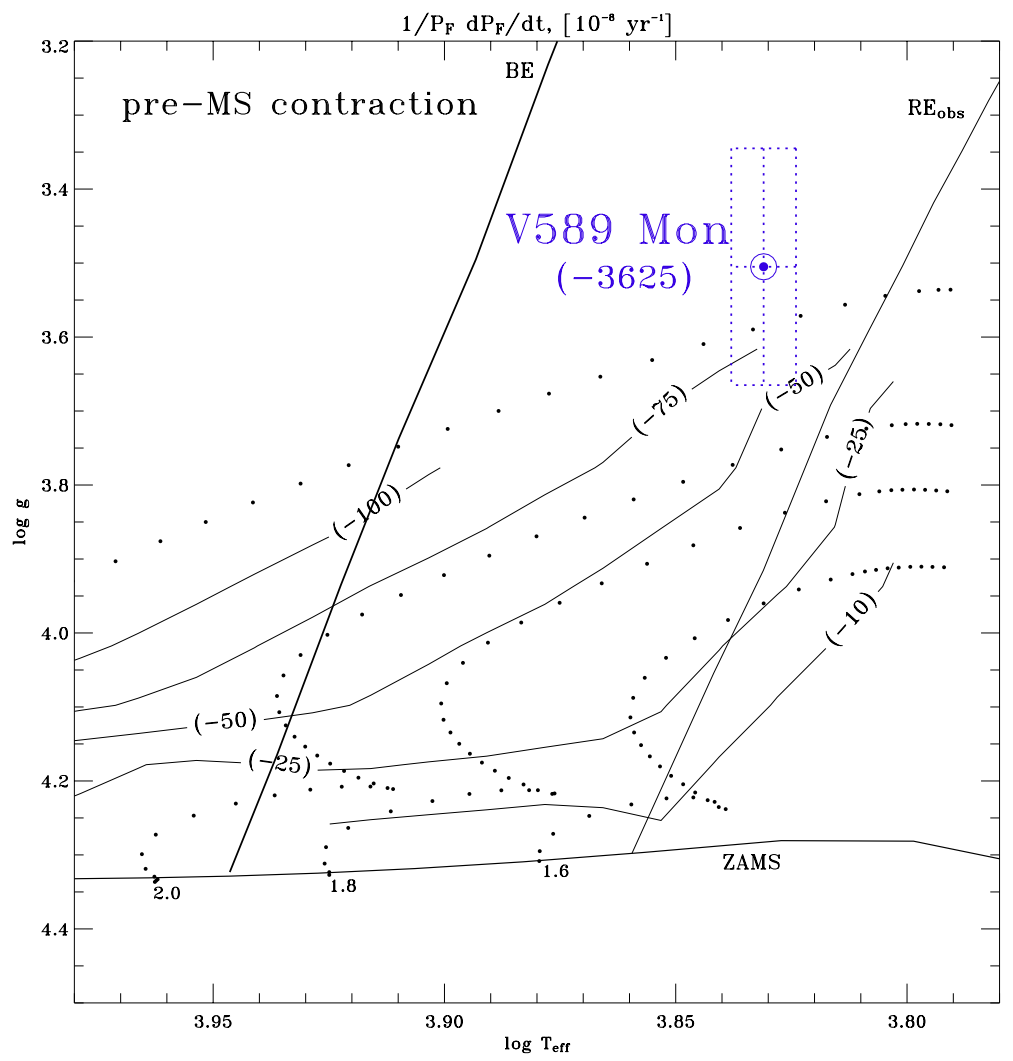

Figure 4. An HR diagram showing the ZAMS, PMS evolutionary tracks (dotted lines), the general blue edge $(\mathrm{BE})$ and the empirical red edge $\left(\mathrm{RE}_{\mathrm{obs}}\right)$ of the classical instability strip and lines of constant period change (solid lines). The position of V 589 Mon according to its $T_{\text {eff }}$ and $\log g$ is marked (dot) including the errors (box). The star shows an expected period change of -75 to $-100 \cdot 10^{-8} \mathrm{yr}^{-1}$.

Using data from Breger (1972) together with observations from Peña (2002) performed in 1986, it is possible to detect evolutionary period changes in a PMS star for the first time. As an example, Fig. 4 (Pamyatnykh \& Kallinger 2004) shows the position of V 589 Mon in the HR-diagram, also indicated are the PMS evolutionary tracks (dotted lines, D'Antona \& Mazzitelli 1994), the ZAMS, the general blue edge (BE) and the empirical red edge $\left(\mathrm{RE}_{\mathrm{obs}}\right)$ of the classical instability strip and lines of constant period change (solid lines). According to its position V 589 Mon is expected to show a period change of -75 to $-100 \cdot 10^{-8} \mathrm{yr}^{-1}$ within the given error bars. But our measurements yield a period change of $-3625 \cdot 10^{-8} \mathrm{yr}^{-1}$, indicating that stellar evolution in this phase is much faster than expected. More data are needed to investigate such period changes in greater detail.

\section{Conclusions}

From the 24 PMS pulsating stars now known, nine are members of young open clusters, while the others are Herbig Ae field stars. All PMS pulsators lie within the boundaries of the classical instability strip, but a gap seems to exist towards the red edge. It is essential to investigate whether this lack of stars is due to poor-number statistics, an overlap with the $\mathrm{T}$ Tauri domain or an evolutionary effect. A larger number of known PMS 
pulsators would help to settle this question, but also permit investigations to better define the boundaries of the PMS instability strip observationally and especially investigate a possible difference in the classical instability region.

Period changes due to evolutionary effects need to be studied in more detail to find whether stellar evolution is indeed a factor 40 faster than expected by theory.

\section{Acknowledgements}

This project was supported by the Austrian Fonds zur Förderung der wissenschaftlichen Forschung (P14984). Fourier Analysis was performed using the program PERIOD98 written by M. Sperl (1998). Use was made of the WEBDA database, operated at the Institute of Astronomy of the University of Lausanne.

\section{References}

Amado P.J., Moya A., Suárez J.C., Martin-Ruiz S., Garrido R., Rodriguez E., Catala C., Goupil M.J. 2004, MNRAS, 352, L11

Breger M. 1972, ApJ, 171, 539

D'Antona F., Mazzitelli I. 1994, ApJS, 90, 467

Kenyon S.J., Hartmann L. 1995, ApJS, 101, 117

Marconi M. 2004, priv. communication

Pamyatnykh A. \& Kallinger T. 2004, priv. communication

Peña J. H., Peniche R., Cervantes F., Parrao L. 2002, RMxAA, 38, 31

Thé P.S., Hageman T., Westerlund B.E., Tjin A Djie H.R.E. 1985, A\&\&A, 151, 391

van den Ancker M.E., Thé P.S., de Winter D. 2000, A\&BA, 362, 580

Zwintz K., Marconi M., Reegen P., Weiss W.W. 2004, MNRAS, in press

\section{Discussion}

NoELs: What is the error bar on the effective temperature? Could it be a way to populate the region of "missing" stars?

ZwINTZ: The accuracy in effective temperature for these stars is $\pm 150 \mathrm{~K}$. Only with the discovery of cooler PMS pulsators can the lack of stars towards the red edge of the instability region be explained.

MiCHEL: Do we have an idea of the distribution of rotational velocities for these objects?

ZWINTZ: Not for all of the stars rotational velocities and/or $v \sin i$ values are available, but generally young stars are believed to be fast rotators. 